\title{
Solutal Marangoni flow as the cause of ring stains from drying salty colloidal drops
}

\author{
Alvaro Marin, ${ }^{1, *}$ Stefan Karpitschka, ${ }^{2}$ Diego Noguera-Marín, ${ }^{3}$ Miguel A. Cabrerizo-Vílchez, ${ }^{3}$ \\ Massimiliano Rossi, ${ }^{4}$ Christian J. Kähler, ${ }^{4}$ and Miguel A. Rodríguez Valverde ${ }^{3}$ \\ ${ }^{1}$ Physics of Fluids Group, Max Planck Center for Complex Fluid Dynamics, University of Twente, \\ Enschede, The Netherlands \\ ${ }^{2}$ Max-Planck Institute for Dynamics and Self-Organization (MPIDS), D-37077 Göttingen, Germany \\ ${ }^{3}$ Biocolloid and Fluid Physics Group, University of Granada, Spain \\ ${ }^{4}$ Institut für Strömungsmechanik und Aerodynamik, Universität der Bundeswehr München, Germany
}

(Received 18 September 2018; published 29 April 2019)

\begin{abstract}
Evaporating salty droplets are ubiquitous in nature, in our home and in the laboratory. Interestingly, the transport processes in such apparently simple systems differ strongly from evaporating "freshwater" droplets since convection is partly inverted due to Marangoni stresses. Such an effect has crucial consequences to the salt crystallization process and to the deposits left behind. In this work we show unprecedented measurements that, not only confirm clearly the flow inversion, but also elucidate their impact on the distribution of nonvolatile solutes. Contrary to what has been often reported in the literature, such a flow reversal does not prevent the formation of ring-shaped stains: particles accumulate at the contact line driven solely by the interfacial flow. We can therefore conclude that the classical "coffee-stain effect" is not the only mechanism that can generate ring-shaped stains in evaporating droplets.
\end{abstract}

DOI: 10.1103/PhysRevFluids.4.041601

Evaporating sessile liquid droplets are ubiquitous in our daily environment and in many industrial applications, for instance in evaporative self-assembly techniques [1]. Consequently, evaporating sessile droplets have been intensively studied recently, in particular after the seminal work of Deegan et al. [2]. In most applications, the droplet does not only contain a dispersion of particles in a pure solvent, but also many other, soluble components. If such components are surface-active, the transport processes inside the droplet can be drastically affected. That has been shown for bulk mixtures [3-6] and surfactant solutions [7-9].

The effect of nonvolatile solutes, like salts, is paramount since they are part of most liquids in biomedical applications to match the ionic strength and the $p \mathrm{H}$ of the human body. Salty droplets are of particular interest also in the pharmaceutical industry where controlled crystallization is crucial for drug formulation [10]. Salt solutions in contact with metallic surfaces provoke corrosion, and although ubiquitous, the underlying mechanisms remain poorly understood $[11,12]$. In most cases of salty droplet evaporation, the residual components enrich near the contact line. Therefore, crystallization and growth of the final deposit typically nucleates at the contact line [12-14]. Recent artwork of the photographer Maurice Mikkers displayed micrographs of dried teardrops, itemized by the emotional state under which the tears were shed [15].

Peripheral salt enrichment leads to Marangoni convection, which in turn affects the solute distribution [16]. Chaotropic salts act as "antisurfactants" [17] and increase the surface tension of

\footnotetext{
*Corresponding author: a.marin@utwente.nl
} 


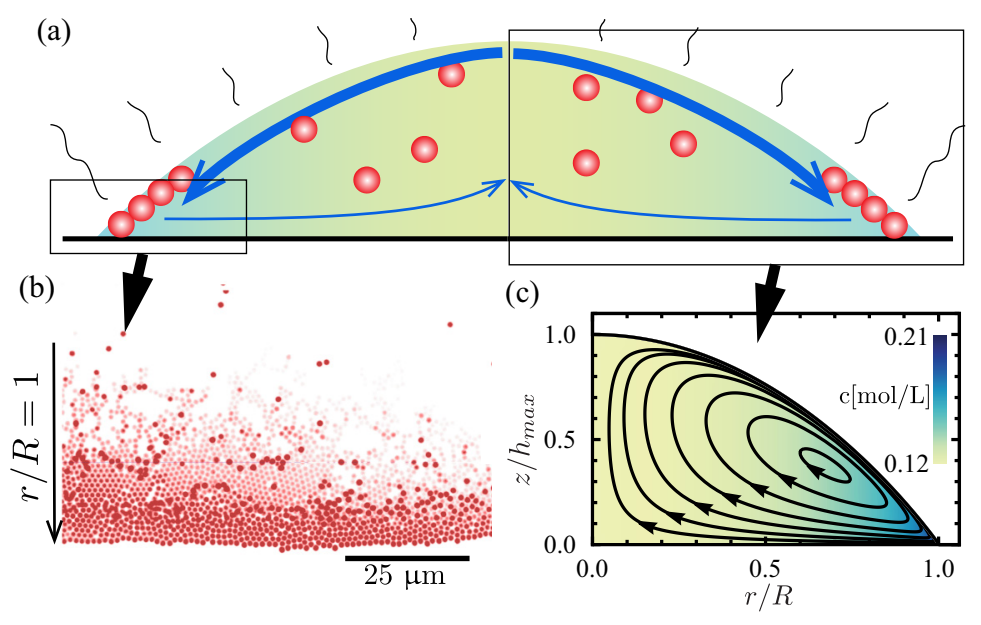

FIG. 1. (a) Sketch of a sessile droplet containing a nonsaturated aqueous saline solution and a dilute dispersion of polymer microparticles. The blue lines illustrate the direction of the flow within the droplet. Note that a large portion of the liquid (close to the substrate) flows in opposite direction as in the classical "coffee-stain effect." (b) Particles reach the contact line along the liquid-gas interface, forming a ring-shaped stain. Here the contact line is defined as $r / R=1$, where $R$ is the droplet radius and $r$ the radial distance from the center. (c) The inverted vortical flow profile is shown through the computed streamlines, which is also measured experimentally via particle tracking velocimetry [see Fig. 3(a)].

water. Thus the associated Marangoni convection is directed toward the contact line. Under pinned contact line conditions, volume conservation then leads to a reversal of the capillary flow near the substrate, which is opposite to the case of pure water. The existence of such a nonmonotonic radial flow patterns provokes a simple yet nontrivial question: Will the deposits be ring shaped as in the classical coffee-stain effect?

In this Rapid Communication we present experimental and numerical evidence for soluteinduced Marangoni stresses that dominate the whole flow within an evaporating droplet; we also demonstrate the existence of a new type of ring-shaped stain that, due to a surface-driven Marangoni flow, assembles along the liquid-vapor interface. This is in stark contrast to the commmonly observed assembly in bulk for "freshwater" droplets [2]. To show this unambiguously we first observe and quantify the growth of the particle deposits in evaporating water droplets for different salt concentrations using confocal imaging. Secondly, we measure the full three-dimensional (3D) flow inside the evaporating salty droplet using 3D particle tracking velocimetry. Finally, we compare the experimental results with numerical simulations that capture the solvent evaporation, the evaporation-induced liquid flow, and the quasiequilibrium liquid-gas interface.

The system that we are considering is summarized in Fig. 1: a sessile water droplet of $1 \mu 1$ (initial contact angle $\sim 15^{\circ}$ ) containing a sodium chloride $(\mathrm{NaCl})$ solution is deposited on a glass slide and evaporates at room temperature and an ambient relative humidity of $40 \%$ [15]. We used initial concentrations of $C_{o}=0,1,2,5$, and $10 \mathrm{mM}$, far from saturation. In order to study how the deposit structure is formed, we use confocal microscopy to observe a thin optical plane close to the contact line and to the glass substrate. A small amount of charged-stabilized polystyrene fluorescent particles are introduced in the droplet (particle diameter $1.11 \mu \mathrm{m}$, microparticles $\mathrm{GmbH}$, below $0.001 \% \mathrm{w} / \mathrm{w})[20]$.

Figure 2(a) shows snapshots of the contact line region in the last stage of solvent evaporation, at identical times after droplet deposition. The number of initially dispersed particles was kept constant for all experiments; only the initial salt concentration $C_{o}$ was increased. Unambiguously, the amount of particles that aggregate at the contact line increases with $C_{o}$. On panel (b), we quantified the number of particles per unit of wet area against time (see also Ref. [21] for details on the method). 


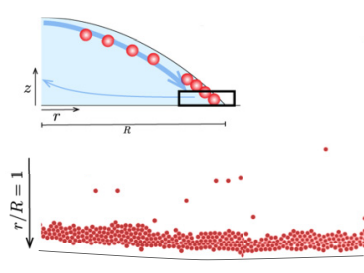

(a)

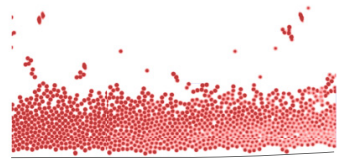

$0 \mathrm{mM} \quad 1 \mathrm{mM}$
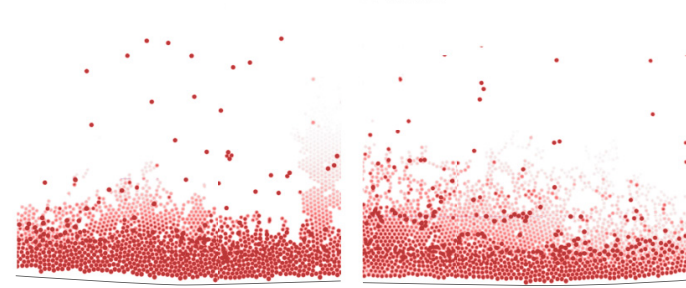

$2 \mathrm{mM}$

$5 \mathrm{mM}$

$25 \mu \mathrm{m}$

(b)

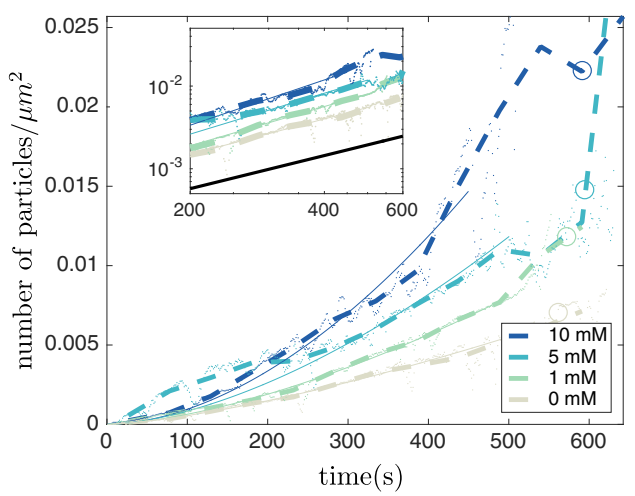

FIG. 2. (a) Images close to the contact line (black line) depicting the particle accumulation in the final stage of drop evaporation for increasing $\mathrm{NaCl}$ concentrations. The droplet radius $R$ for all experiments is $1 \mathrm{~mm}$ and the total evaporation time about $1000 \mathrm{~s}$. All images are taken using a confocal microscope in a $z$ position close to the glass slide. Filled red circles are fluorescent particles accumulated at the bottom substrate; dimmer ones are located in higher layers. (b) Number of particles near the contact line per unit of wet area against the time for different solute concentration. The dashed lines show the binned data. Circles depict the instants in which images from Fig. 2(a) were taken. Inset: Final values of normalized particle increment in terms of $\mathrm{NaCl}$ concentration. Black continuous line corresponds to the stain growth $N \propto t^{1.33}$, reported by Deegan et al. [2] for comparison.

In a $5 \mathrm{mM}$ salt solution, aggregation is accelerated by a factor of 2 as compared to pure water. In the light of Deegan's classical results [22], we fit simple power laws to the amount of particles per unit area $N \sim t^{\alpha}$. Without salt we obtain $\alpha \sim 1.4$ which compares well with [22]. Prefactor and exponent increase with the amount of salt in the system, reaching $\alpha \sim 1.8$ for $10 \mathrm{mM}$ [inset in Fig. 2(b)], indicating the salt-enhanced accumulation.

The mechanism of this enhanced ring-stain formation is revealed by the shading and the defocusing of the particles in Fig. 2(a): lighter tones correspond to particles that are more distant from the focal plane and thus from the substrate. At high salt concentrations $\left(C_{o} \geqslant 2 \mathrm{mM}\right)$, most of the particles reach the contact line while following the water-air interface, driven by the solutal Marangoni flow due to salt enrichment. As the initial salt concentration $C_{o}$ is increased further, well-ordered structures of defocused particles come into sight, i.e., at the liquid-air interface. At the same time, fewer particles appear in the focal plane, i.e., just above the liquid substrate. The observation of the deposit formation yields two surprising effects: (1) an increase in the particle flux with the amount of salt and (2) the arrival of the particles mostly along the interface and not through the bulk, as is typically observed in "freshwater" droplets.

Since an explanation of this effect must be deeply connected to the convection in the droplet, we next measure the three-dimensional velocity field using astigmatism particle tracking velocimetry (APTV) [18,19]: a single-camera technique that yields all velocity components for each tracer particle in space over time [20]. The experimental results are shown in Fig. 3(a) (Fig. S1 in the Supplemental Material explicitly shows particle trajectories from the measurements) for a droplet of initial volume $0.8 \mu \mathrm{l}$, initial salt $(\mathrm{NaCl})$ concentration of $100 \mathrm{mM}$, and initial contact angle of $40^{\circ}$. The experimental results clearly show the "inverted" flow profile inside the evaporating water saline droplet consisting of a vortical flow structure that drives the liquid in the bulk toward the droplet's center, and toward the contact line at the liquid-air interface. Consequently, most of the 
(a)

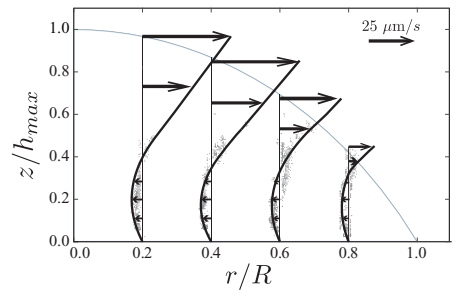

(b)

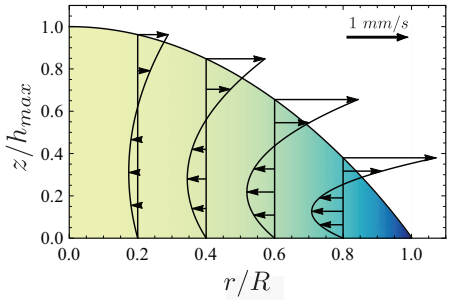

(c)

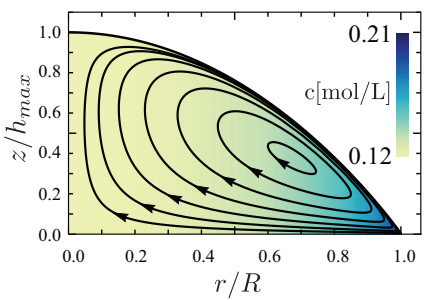

FIG. 3. (a) Experimentally obtained flow profile in an evaporating droplet obtained by a 3D particle tracking (APTV $[18,19]$ ). (b) Velocity profile and (c) flow streamlines in an evaporating droplet with an initial $\mathrm{NaCl}$ concentration of $100 \mathrm{mM}$ obtained by solving the Stokes flow inside the droplet in the lubrication approximation. The instant shown corresponds to $50 \%$ of the total evaporation time. The experimental conditions are identical as those in the simulations shown in (b) and (c). Note that in both simulations and experiments the interfacial flow toward the contact line dominates the flow in the droplet.

particles reach the contact line through the droplet's surface, and not through the bulk as happens with pure water solutions and in the classical coffee-stain effect.

Contrary to surfactant solutions, chaotropic electrolytes increase the surface tensions of water, as is well known from classical thermodynamics [23-25]. For $\mathrm{NaCl}$, this effect quantifies as $\sim 1.5 \mathrm{mN} / \mathrm{m}$ per mol NaCl per liter of water [26]. Therefore, it is expected that an accumulation of salt at the contact line would generate a surface tension gradient toward the droplet's border. The gradient is clearly strong enough to overcome the evaporation-driven bulk flow or any additional thermal Marangoni convection. Due to the pinning condition at the contact line, the outward directed Marangoni flow must be compensated by an "inverted" capillary flow toward the center of the droplet.

To get a deeper insight into the evaporation process, the resultant flow, and the possible particle accumulation mechanisms, we performed numerical simulations for the advection-diffusion problem in the droplet in the lubrication approximation on a radially symmetric domain. The fluid flow is coupled to the diffusion problem in the gas phase via Raoult's law and a Stefan condition. The salt is introduced through a compositional variable that diffuses, is advected with the bulk flow, and alters the physical properties of the liquid. Most importantly, it alters surface tension and vapor pressure, for which we have used literature values [27-29]. One important limitation of the model is that crystallization is not explicitly modeled. Although saturation conditions are not reached in the simulations, this is typically the case during the latest stage of the experiments, but only after the initial particle deposit structure could be observed. The results of the numerical simulations are shown in Figs. 3 and 4. In Figs. 3(b) and 3(c) it can be seen that the quality of the resultant flow structure is identical as compared to the experiments [Fig. 3(a)]. The simulations show a strong accumulation of salt at the contact line due to the stronger local evaporation [Fig. 4(b)], and consequently, a strong surface tension gradient directed toward the contact line. The surface tension gradient generates the Marangoni flow observed, which dominates the flow within the droplet (Fig. 3). Note that the singularity in the evaporative flux at the contact line is regularized only at a (sub-)micron length scale for millimetric droplets [Fig. 4(c)] [22,30]. Thus the evaporation rate peaks sharply at the contact line, and peak height and width change by only about half a decade over time, due to salt enrichment.

Despite the qualitative analogy in the flow profiles computed numerically and measured experimentally, a significant quantitative disagreement is found in the value of the velocities (explicit in Fig. 3), in which simulations predict much stronger Marangoni convection. Numerical simulations have been repeated using different methods (finite elements and finite volume schemes, using various software packages) to exclude technical issues like the level of approximation or discretization effects [31]. Experiments have been replicated in different laboratories, with different 
(a)

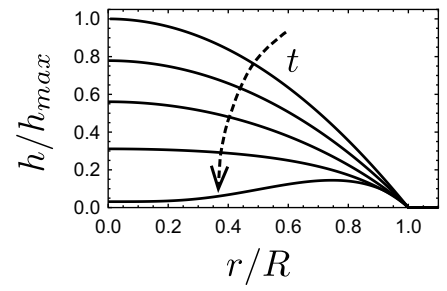

(b)

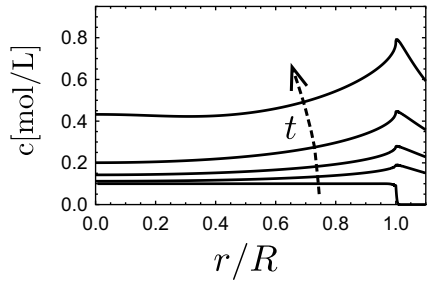

(c)

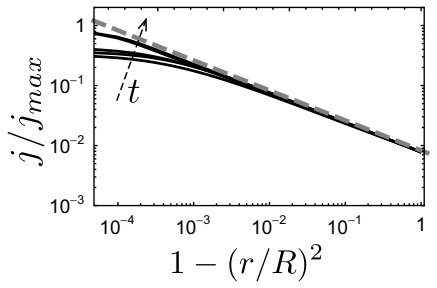

FIG. 4. Results from the numerical simulations on the evaporating salty droplets. The plots correspond to different stages in the evaporation of a droplet containing $\mathrm{NaCl}$ at a concentration of $100 \mathrm{mM}$, initial contact angle of $20^{\circ}$, and radius of $1 \mathrm{~mm}$. The different lines correspond to $0,20,40,60$, and $80 \%$ of the total evaporation time: (a) droplet interface $\mathrm{h}(\mathrm{r})$ in time, (b) salt concentration $c$ in mol/l (c), and normalized evaporative flux $j / j_{\max }$, which becomes singular as the contact line is approached $(r / R \rightarrow 1)$ [2].

equipment and samples - including different particle sizes and types- always obtaining the same results. It is interesting to note that a similar quantitative disagreement is found in water droplets in thermal Marangoni flows [7] and with certain bicomponent systems [32]. Given the consistency of the experimental results, we do not invoke here the commonly accepted explanation through surface active trace impurities [33]. It could be argued that colloidal particles are not good flow tracers for interfacial flows due to complex particle-interface interactions. In any case, although a physical explanation for the quantitative disagreement found in Marangoni-dominated evaporating droplets is still lacking, our simulations confirm the nature of the interfacial flow observed in the experiments.

From the information gained in experiments and simulations, we can conclude that for salt concentrations above $2 \mathrm{mM}$ particles arriving at the contact line are either adsorbed at the liquid-air interface or stabilized very close to it. The increase of initial salt concentration (within the range 0 $200 \mathrm{mM}$ ) also increases the maximum concentration gradient achievable, increasing the interfacial velocity and therefore the flux of particles toward the contact line [Fig. 2(b)].

Particle accumulation at interface region is an important step in the process. Although our experimental method is not able to obtain the precise particle position with respect to the interface, it is evident in our own measurements that particles tend to accumulate in its vicinity (see Supplemental Material). Others have observed a similar behavior with particles in salt-free solutions [34,35] or in surfactant solutions [36]. Recently, Kang et al. [37] proposed a mechanism for the formation of ring-shaped stains in water droplets solely based on the capture of particles at the receding interface and subsequently transported along the interface until they are deposited near the contact line. However, sessile water droplets evaporating in atmospheric conditions experience a weak but persistent thermal Marangoni flow which is directed away from the contact line $[7,38]$. Thus a thermal Marangoni flow of such orientation would actually prevent ring-stain formation [16]. The mechanism proposed by Kang et al. [37] for ring-stain formation would be valid only when there is a dominant outward-directed interfacial flow, as in the case of salty droplets.

Recently, the formation of thin salt shells along the interface of evaporating saline sessile droplets has been reported in different systems [39,40], which would be easily explained by the presence of the interfacial flow reported in our work. Salt-enriched liquid enriches the interface closer to the contact line, favoring interfacial crystallization. Note that similar flow inversion has been observed in evaporating ethanol-water mixtures [41], in which the enrichment of water provokes a positive surface tension gradient toward the contact line. This principle also holds for Ouzo droplets [42]; however, phase separation effects dominate later stages of the evaporation process, causing nontrivial deposit patterns.

In conclusion, we have shown that ring-shaped stains from evaporating antisurfactant [17] droplets do not form via the classical, capillary flow based mechanism [2], but via solutal Marangoni 
flows and interfacial aggregation. This is not only the case for sodium chloride solutions but also for chaotropic salt solutions in general, with unexpected consequences in systems involving sessile droplets of salt solutions.

A.M., M.R., and C.K. acknowledge financial support by the Deutsche Forschungsgemeinschaft under Projects No. KA 1808/12-2 and No. KA 1808/22-1. A.M. and S.K. are grateful to Christian Diddens for several discussions on the topic. M.A.R.V. and M.C.V. acknowledge financial support by the MINECO Grants No. MAT2014-60615-R and No. MAT2017-82182-R. S.K. acknowledges financial support from the Max Planck Center for Complex Fluid Dynamics.

[1] W. Han and Z. Lin, Learning from "coffee rings": Ordered structures enabled by controlled evaporative self-assembly, Angew. Chem., Int. Ed. 51, 1534 (2011).

[2] R. D. Deegan, O. Bakajin, T. F. Dupont, G. Huber, S. R. Nagel, and T. A. Witten, Capillary flow as the cause of ring stains from dried liquid drops, Nature (London) 389, 827 (1997).

[3] S. Karpitschka, F. Liebig, and H. Riegler, Marangoni contraction of evaporating sessile droplets of binary mixtures, Langmuir 33, 4682 (2017).

[4] S. Karpitschka and H. Riegler, Noncoalescence of Sessile Drops from Different but Miscible Liquids: Hydrodynamic Analysis of the Twin Drop Contour as a Self-Stabilizing Traveling Wave, Phys. Rev. Lett. 109, 066103 (2012).

[5] N. J. Cira, A. Benusiglio, and M. Prakash, Vapour-mediated sensing and motility in two-component droplets, Nature (London) 519, 446 (2015).

[6] Y. Li, P. Lv, C. Diddens, H. Tan, H. Wijshoff, M. Versluis, and D. Lohse, Evaporation-Triggered Segregation of Sessile Binary Droplets, Phys. Rev. Lett. 120, 224501 (2018).

[7] A. Marin, R. Liepelt, M. Rossi, and C. J. Kähler, Surfactant-driven flow transitions in evaporating droplets, Soft Matter 12, 1593 (2016).

[8] W. Sempels, R. De Dier, H. Mizuno, J. Hofkens, and J. Vermant, Auto-production of biosurfactants reverses the coffee ring effect in a bacterial system, Nat. Commun. 4, 1757 (2013).

[9] M. A. Bruning, M. Costalonga, S. Karpitschka, and J. H. Snoeijer, Delayed coalescence of surfactant containing sessile droplets, Phys. Rev. Fluids 3, 073605 (2018).

[10] B. Zheng, L. S. Roach, and R. F. Ismagilov, Screening of protein crystallization conditions on a microfluidic chip using nanoliter-size droplets, J. Am. Chem. Soc. 125, 11170 (2003).

[11] J. Li, R. Wu, Z. Jing, L. Yan, F. Zha, and Z. Lei, One-step spray-coating process for the fabrication of colorful superhydrophobic coatings with excellent corrosion resistance, Langmuir 31, 10702 (2015).

[12] V. Soulie, S. Karpitschka, F. Lequien, P. Prene, T. Zemb, H. Moehwald, and H. Riegler, The evaporation behavior of sessile droplets from aqueous saline solutions, Phys. Chem. Chem. Phys. 17, 22296 (2015).

[13] N. Shahidzadeh-Bonn, S. Rafaï, D. Bonn, and G. Wegdam, Salt crystallization during evaporation: Impact of interfacial properties, Langmuir 24, 8599 (2008).

[14] N. Shahidzadeh, M. F. L. Schut, J. Desarnaud, M. Prat, and D. Bonn, Salt stains from evaporating droplets, Sci. Rep. 5, 1 (2015).

[15] See http://www.imaginariumoftears.com

[16] H. Kim, F. Boulogne, E. Um, I. Jacobi, E. Button, and H. A. Stone, Controlled Uniform Coating from the Interplay of Marangoni Flows and Surface-Adsorbed Macromolecules, Phys. Rev. Lett. 116, 124501 (2016).

[17] J. J. A. Conn, B. R. Duffy, D. Pritchard, S. K. Wilson, P. J. Halling, and K. Sefiane, Fluid-dynamical model for antisurfactants, Phys. Rev. E 93, 043121 (2016).

[18] C. Cierpka, M. Rossi, R. Segura, and C. J. Kähler, On the calibration of astigmatism particle tracking velocimetry for microflows, Meas. Sci. Technol. 22, 015401 (2011).

[19] M. Rossi and C. J. Kähler, Optimization of astigmatic particle tracking velocimeters, Exp. Fluids 55, 1809 (2014). 
[20] See Supplemental Material at http://link.aps.org/supplemental/10.1103/PhysRevFluids.4.041601 for more details on the experimental conditions and on the astigmatism particle tracking velocimetry method.

[21] D. Noguera-Marín, C. L. Moraila-Martínez, M. A. Cabrerizo-Vílchez, and M. A. Rodríguez-Valverde, In-plane particle counting at contact lines of evaporating colloidal drops: Effect of the particle electric charge, Soft Matter 11, 987 (2015).

[22] R. D. Deegan, O. Bakajin, T. F. Dupont, G. Huber, S. R. Nagel, and T. A. Witten, Contact line deposits in an evaporating drop, Phys. Rev. E 62, 756 (2000).

[23] E. Hückel, Zur theorie der elektrolyte, Ergebnisse der Exakten Naturwissenschaften (Springer, Berlin, Heidelberg, 1924), pp. 199-276.

[24] L. Onsager and N. N. T. Samaras, The surface tension of Debye-Hückel electrolytes, J. Chem. Phys. 2, 528 (1934).

[25] Y. Levin and J. E. Flores-Mena, Surface tension of strong electrolytes, Europhys. Lett. 56, 187 (2001).

[26] N. Matubayasi, H. Matsuo, K. Yamamoto, Shin-ichiro Yamaguchi, and A. Matuzawa, Thermodynamic quantities of surface formation of aqueous electrolyte solutions: I. Aqueous solutions of $\mathrm{NaCl}, \mathrm{MgCl}_{2}$, and $\mathrm{LaCl}_{3}$, J. Colloid Interface Sci. 209, 398 (1999).

[27] Z.-B. Li, Y.-G. Li, and J.-F. Lu, Surface tension model for concentrated electrolyte aqueous solutions by the Pitzer equation, Ind. Eng. Chem. Res. 38, 1133 (1999).

[28] J. I. Partanen and A. K. Covington, Re-evaluation of the thermodynamic activity quantities in aqueous sodium and potassium chloride solutions at $25^{\circ} \mathrm{C}$, J. Chem. Eng. Data 54, 208 (2009).

[29] D. R. Lide, CRC Handbook of Chemistry and Physics (CRC Press, Boca Raton, FL, 2004).

[30] Y. O. Popov, Evaporative deposition patterns: Spatial dimensions of the deposit, Phys. Rev. E 71, 036313 (2005).

[31] Christian Diddens (private communication).

[32] C. Diddens, Detailed finite element method modeling of evaporating multi-component droplets, J. Comput. Phys. 340, 670 (2017).

[33] H. Hu and R. G. Larson, Analysis of the effects of Marangoni stresses on the microflow in an evaporating sessile droplet, Langmuir 21, 3972 (2005).

[34] J. R. Trantum, Z. E. Eagleton, C. A. Patil, J. M. Tucker-Schwartz, M. L. Baglia, M. C. Skala, and F. R. Haselton, Cross-sectional tracking of particle motion in evaporating drops: Flow fields and interfacial accumulation, Langmuir 29, 6221 (2013).

[35] A. I. Abdel-Fattah and M. S. El-Genk, On colloidal particle sorption onto a stagnant air-water interface, Adv. Colloid Interface Sci. 78, 237 (1998).

[36] M. Anyfantakis, Z. Geng, M. Morel, S. Rudiuk, and D. Baigl, Modulation of the coffee-ring effect in particle/surfactant mixtures: The importance of particle-interface interactions, Langmuir 31, 4113 (2015).

[37] S. Jafari Kang, V. Vandadi, J. D. Felske, and H. Masoud, Alternative mechanism for coffee-ring deposition based on active role of free surface, Phys. Rev. E 94, 063104 (2016).

[38] H. Hu and R. G. Larson, Marangoni effect reverses coffee-ring depositions, J. Phys. Chem. B 110, 7090 (2006).

[39] A. Mailleur, C. Pirat, O. Pierre-Louis, and J. Colombani, Hollow Rims from Water Drop Evaporation on Salt Substrates, Phys. Rev. Lett. 121, 214501 (2018).

[40] B. Shin, M.-W. Moon, and H.-Y. Kim, Rings, igloos, and pebbles of salt formed by drying saline drops, Langmuir 30, 12837 (2014).

[41] J. R. E. Christy, Y. Hamamoto, and K. Sefiane, Flow Transition within an Evaporating Binary Mixture Sessile Drop, Phys. Rev. Lett. 106, 205701 (2011).

[42] H. Tan, C. Diddens, P. Lv, J. G. M. Kuerten, X. Zhang, and D. Lohse, Evaporation-triggered microdroplet nucleation and the four life phases of an evaporating Ouzo drop, Proc. Natl. Acad. Sci. USA 113, 8642 (2016). 